\title{
Quiste de uraco infectado en edad infantil
}

\author{
Gimeno Argente V, Domínguez Hinarejos C, Serrano Durbá A, Estornell Moragues F, \\ Martínez Verduch M, García Ibarra F.
}

Servicio de Urología Infantil. Hospital Universitario La Fe. Valencia.

Actas Urol Esp 2006:30(10):1034-1037

\section{RESUMEN}

\section{QUISTE DE URACO INFECTADO EN EDAD INFANTIL}

Los quistes de uraco son anomalías congénitas detectadas con mayor frecuencia en la infancia. Suelen manifestarse cuando se infectan, simulando diversas patologias agudas intraabdominales o pélvicas. Presentamos un caso en una niña de 7 años diagnosticada de quiste de uraco infectado. Analizamos el origen embriológico de esta patología, sus formas de presentación clínica y sus aspectos diagnóstico-terapéuticos.

Palabras clave: Anomalías uracales. Quiste de uraco. Infección.

\section{ABSTRACT}

\section{INFECTED URACHAL CYST DURING CHILDHOOD}

Urachal cysts are congenital anomalies detected predominantly during the childhood. They are often diagnosed when an infection occur mimicking a variety of acute intra-abdominal or pelvic processes. We present a case in a 7-year-old female infant, diagnosed of infected urachal cyst. The embryologic origin of this anomaly, clinical features, diagnosis and treatment are discussed.

Keywords: Urachal anomalies. Urachal cyst. Infection.

$\mathrm{E}^{\prime}$ uraco es una estructura tubular de la línea media que se extiende desde la porción anterior de la cúpula vesical hasta el ombligo. Es un remanente embriológico que deriva de la involución de la alantoides. Esto ocurre después del tercer mes de gestación, iniciándose el descenso de la vejiga hacia la $18^{\text {a }}$ semana, de forma que el cordón umbilical se alarga y los restos de la alantoides se obliteran constituyendo el uraco ${ }^{1,2}$.

Las anomalías uracales tienen como común denominador un fallo en la obliteración de la luz uracal. De ellas, una de las más frecuentes es el quiste de uraco que afecta a uno de cada 5.000 nacimientos ${ }^{3-7}$. Los quistes uracales permanecen habitualmente asintomáticos salvo cuando se complican, siendo la complicación más frecuente la infección.

Presentamos el caso de un quiste de uraco infectado en edad infantil, analizando el diagnóstico y el tratamiento de dicha patología.

\section{CASO CLÍNICO}

Niña de 7 años de edad, sin antecedentes médico-quirúrgicos de interés salvo varios episodios de cistitis aguda (al nacimiento, al año de vida y el último hace 6 meses), que acude al Servicio de Urgencias por dolor abdominal, hematuria macroscópica, febrícula y síndrome miccional irritativo con disuria, polaquiuria y tenesmo vesical de 3 días de evolución, sin mejoría clínica pese al tratamiento antibiótico con Amoxicilina-Clavulánico.

A la exploración física presenta buen estado general y temperatura axilar de $37.2^{\circ} \mathrm{C}$. En la palpación abdominal no se detectan masas ni organomegalias, apreciando leves molestias en la zona periumbilical.

Los datos analíticos sanguíneos son normales salvo leucocitosis de $13.900 \mathrm{cel} / \mathrm{mcl}$ con neutrofilia del $83 \%$, PCR de $51 \mathrm{mg} / 1$ y velocidad de sedimentación globular de $39 \mathrm{~mm} / \mathrm{h}$. El sedimento de 
orina muestra hematuria microscópica, siendo normales el resto de sus parámetros. El urinocultivo que se realiza es negativo.

Se practica una ecografía abdominal que evidencia una lesión quística anfractuosa de 4,3x2 cm, localizada en la pared anterior del abdomen llegando hasta cúpula vesical, con contenido ecogénico en su interior y paredes con vascularización aumentada, sugestiva de quiste de uraco complicado (Fig. 1).

Con la sospecha diagnóstica de quiste de uraco infectado iniciamos tratamiento antibiótico con Fosfomicina, objetivándose mejoría clínica evidente a las pocas horas.

En el seguimiento de la paciente se realiza ecografia abdominal de control, que muestra una masa sólida e irregular, situada encima de la cúpula vesical, de tamaño $1,8 \times 1,2 \mathrm{~cm}$ y en relación con resto del uraco persistente (Fig. 2). De igual forma, realizamos uretrocistografía donde no se aprecia comunicación con la vejiga, ni detectamos la existencia de reflujo vesicoureteral (Fig. 3). Confirmado el diagnóstico de quiste de uraco, programamos la extirpación quirúrgica del mismo. Tras dos semanas con tratamiento antibiótico realizamos laparotomía media infraumbilical-extraperitoneal, procediendo a la exéresis completa del uraco, incluyendo el quiste uracal y un rodete vesical. No hubo complicaciones intraoperatorias y la evolución posterior fue satisfactoria. Actualmente la paciente se encuentra asintomática y en la cistografía practicada al mes de la intervención la vejiga presenta una morfología normal.

\section{DISCUSIÓN}

El uraco es el remanente embriológico de la alantoides, que comunica la cúpula vesical con el ombligo. Entre el $4^{\circ}$ y $5^{\circ}$ mes de gestación se produce el descenso de la vejiga hacia la pelvis, provocando la regresión del uraco que se elonga y estrecha hasta convertirse en un cordón fibromuscular epitelizado que transcurre entre la fascia transversalis y el peritoneo parietal, alcanzando en el adulto una longitud que oscila entre 3 y $10 \mathrm{~cm}$ y entre 8 y $10 \mathrm{~mm}$ de diámetro ${ }^{2}$.

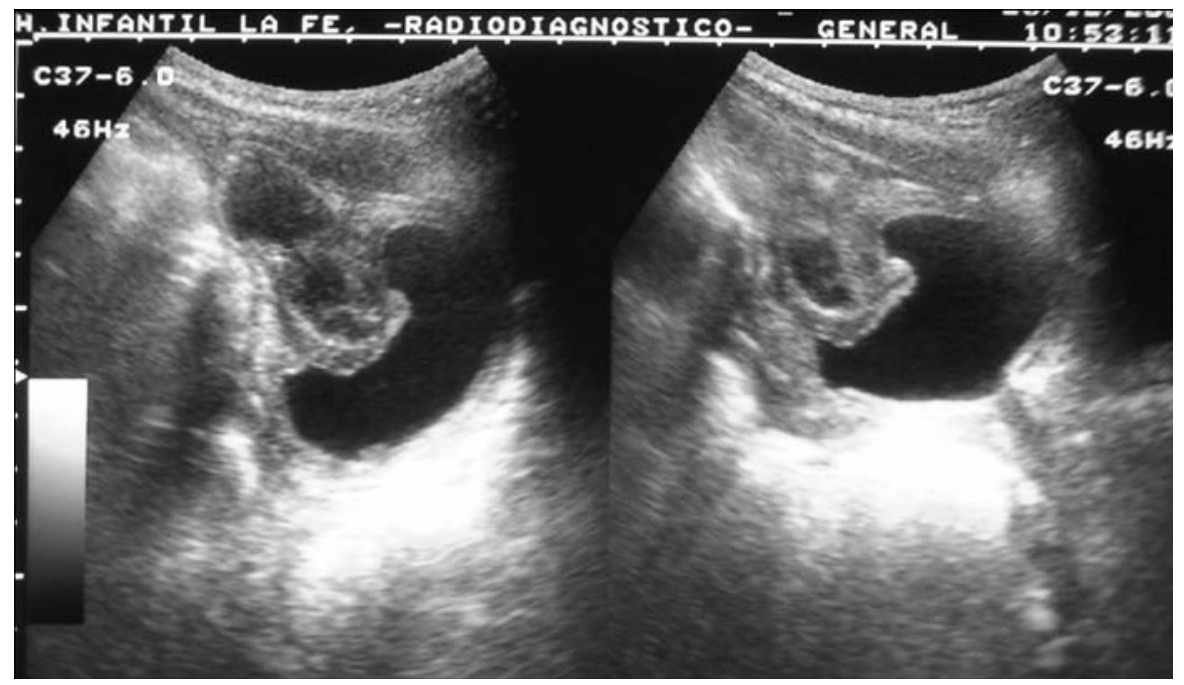

FIGURA 1. Ecografía vesical: Lesión quística irregular, de tamaño aproximado $4 \times 2$ $\mathrm{cm}$, de ecogenicidad heterogénea, situada en pared anterior del abdomen contactando con la cúpula vesical.

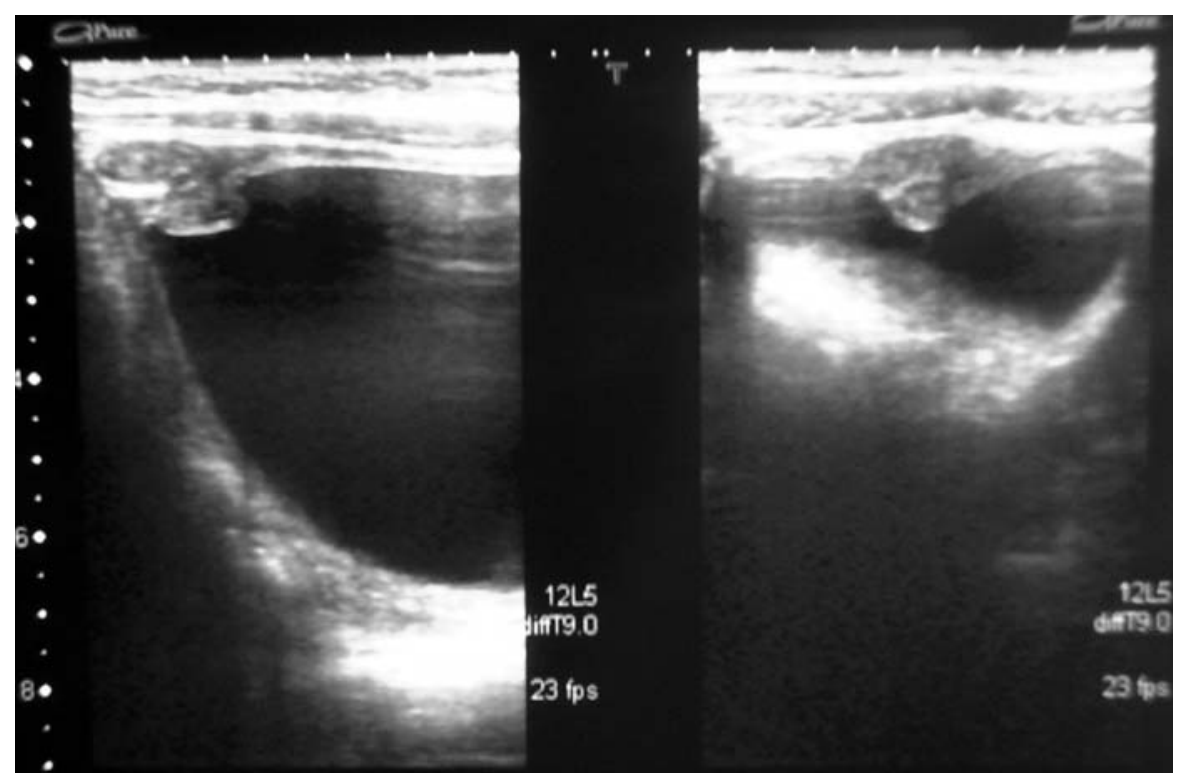

FIGURA 2. Imágenes ecográficas vesicales que muestran una masa sólida e irregular por encima de la cúpula vesical, en relación con resto del uraco persistente. 


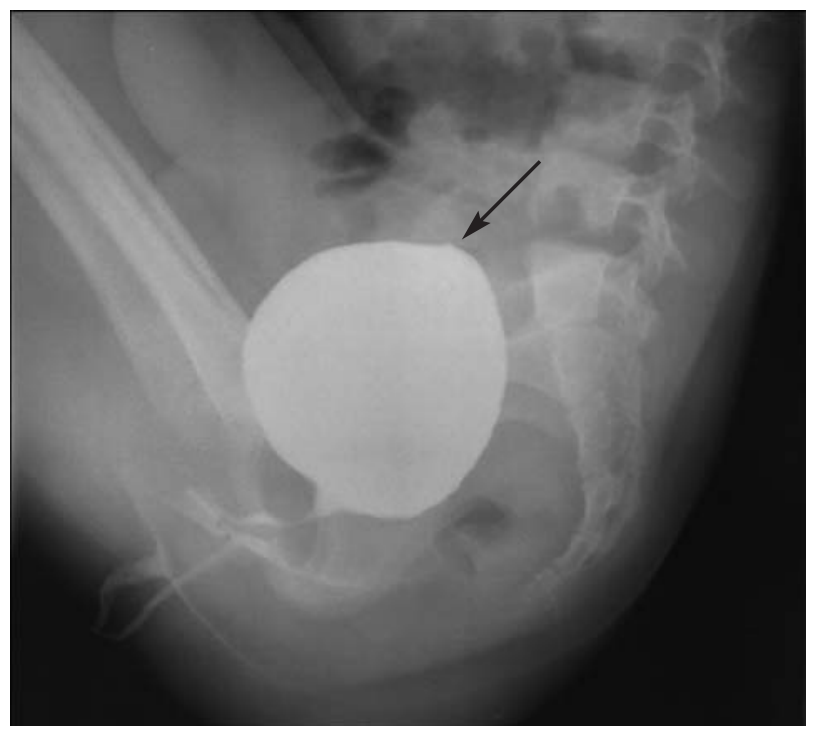

FIGURA 3. Imagen de uretrocistografia donde podemos apreciar el resalte que provoca el quiste de uraco en la cúpula vesical (flecha). No se observa comunicación con la vejiga, ni reflujo vesicoureteral.

Histológicamente consta de un revestimiento interior de epitelio transicional (en el 70\% de los casos) o cuboideo (en el 30\% restante), rodeado por una capa submucosa de tejido conectivo, y una capa de músculo liso externa que tiene continuidad con el detrusor ${ }^{8}$.

En el momento del nacimiento, el uraco suele estar obliterado, manteniéndose permeable únicamente en el $2 \%$ de los adultos ${ }^{1}$. Los defectos de obliteración del uraco dan lugar a distintas anomalías, resultantes de las diferentes comunicaciones que se establecen entre vejiga, uraco y ombligo $^{9}$.

Las variedades de anomalias uracales son las siguientes: persistencia del uraco (supone alrededor del 50\% de todas las anomalías uracales, detectándose un uraco permeable en todo su trayecto), quiste uracal (representa el 30\% de estos defectos congénitos), seno uracal (aparece en el $15 \%$ de casos y consiste en la ausencia de cierre de la porción apical del uraco, que queda comunicado con el ombligo) y divertículo de uraco (la porción del uraco próxima a la vejiga permanece permeable, constituyendo entre el 3 y $5 \%$ de esta patologia) $2,10,11$.

El quiste uracal se produce por una obliteración parcial del uraco, al cerrarse los extremos cutáneo y vesical, quedando una cavidad quística intermedia de contenido líquido seroso con células descamadas. Su incidencia es escasa, estimándose en uno cada 5.000 nacimientos, con una proporción de 3:1 a favor de los varones ${ }^{5,12}$.

La mayoría de quistes uracales son de pequeño tamaño y asintomáticos, por lo que permanecen indetectables hasta que debutan clínicamente con sus complicaciones. Estas potenciales complicaciones incluyen crecimiento progresivo, formación de cálculos, infección, sangrado intraquístico, rotura intraperitoneal, fístula a intestino, obstrucción intestinal y del tracto urinario, desarrollo de enfermedad de Reiter y malignización ${ }^{4,5}$. De ellas, la más común es la infección provocada por la migración de gérmenes desde el ombligo o la vejiga, aunque también se ha implicado las vías hematógena y/o linfática. Entre los patógenos responsables de esta infección, se han aislado gérmenes aerobios ${ }^{13}$, anaerobios y bacilos gram positivos intestinales, bacilos gram negativos de origen urinario ${ }^{14}$ e incluso hongos. La mayoría de autores coinciden al indicar que el Staphylococcus Aureus es el microorganismo que con más frecuencia provoca infección del quiste de uraco $^{12,15,16}$.

El diagnóstico de un quiste infectado es clínico, ya que suele manifestarse como fiebre, dolor abdominal inferior alrededor de la línea media, clínica miccional con o sin infección urinaria y en ocasiones masa palpable suprapúbica. En el diagnóstico diferencial de los quistes de uraco infectados debemos considerar patologias como enfermedad pélvica inflamatoria, apendicitis aguda, cistitis, enfermedad inflamatoria intestinal, perforación visceral, hernia umbilical estrangulada, absceso pélvico o intraabdominal y divertículo de Meckel $^{12}$.

Entre las pruebas complementarias útiles para el diagnóstico de esta entidad, destaca la ecografía como prueba de imagen ideal al tratarse de una patología quística, extraperitoneal y en relación con la vejiga ${ }^{4,5,15}$. El porcentaje de éxito diagnóstico de la ecografía oscila entre el 75\% y el 100\% para esta patología, según series publicadas $^{12}$. El TC no suele ser necesario para su diagnóstico, sin embargo puede ser efectivo para diferenciar esta patología de otras de origen intraabdominal como la apendicitis aguda, la diverticulitis de Meckel o la torsión de ovarios ${ }^{4,17}$. Otras pruebas como la uretrocistografia son de 
controvertida utilidad. Así, para algunos autores esta prueba no aporta demasiado en el diagnóstico de esta patología, sin embargo otros defienden su utilidad a la hora de evaluar la comunicación entre vejiga y uraco, al tiempo que recuerdan la asociación entre anomalias genitourinarias como el reflujo vesicoureteral y la enfermedad quística uracal, ya que en algunos casos la persistencia del uraco podría estar en relación con obstrucción infravesical ${ }^{18}$.

El tratamiento del quiste de uraco dependerá de si presenta complicaciones asociadas o no. Los quistes de uraco no infectados pueden ser tratados con éxito en un sólo acto quirúrgico, realizando exéresis completa de la lesión acompañado de un pequeño rodete de la cúpula vesical, ya que el drenaje simple del quiste se asocia a un $30 \%$ de recurrencias ${ }^{1,8,12}$. Otro motivo que justifica la extirpación completa del remanente uracal es la posibilidad de degeneración maligna tardía a adenocarcinoma, sarcoma o carcinoma de células transicionales que ha sido observada en una proporción de 1/5.000.0004,14,19.

En caso de infección la opción preferida por la mayoría de autores, al igual que por nosotros, es el tratamiento antibiótico "enfriando" el proceso para procurar su confinamiento ${ }^{9}$. En ocasiones a este tratamiento médico es necesario asociar el drenaje del quiste. Con ello conseguimos mejorar el cuadro inflamatorio lo que facilitará el procedimiento quirúrgico que realizaremos en un segundo tiempo, a la vez que disminuye el número de complicaciones postoperatorias ${ }^{4}$.

\section{REFERENCIAS}

1. Jalón Monzón A, Rodríguez Faba O, García Rodríguez J, Díaz Sotres M, Fernández Gómez J, Escaf Barmadan S et al. Actinomicosis uracal: a propósito de un caso. Actas Urol Esp. 2002;26(7):519-522.

2. Yu JS, Kim KW, Lee HJ, Lee YJ, Yoon CS, Kim MJ. Urachal remnant diseases: spectrum of CT and US findings. Radiographics. 2001;21(2):451-461.

3. McCollum MO, MacNeily AE, Blair GK. Surgical implications of urachal remnants: presentation and management J Pediatr Surg. 2003;38(5):798-803.

4. Gómez Díaz ME, Tornero Ruiz JI, Caffaratti Sfulcini J, Garat Barredo JM. Quistes uracales en la infancia. Arch Esp Urol. 2003;56(3):300-302.
5. Minevich E, Wacksman J, Lewis AG, Bukowski TP, Sheldon CA. The infected urachal cyst: primary excision versus a staged approach. J Urol. 1997;157(5):1869-1872.

6. Ogbevoen JO, Jaffe DM, Langer JC. Intraperitoneal rupture of an infected urachal cyst: a rare cause of peritonitis in children. Pediatr Emerg Care. 1996;12(1):41-43.

7. Goldman IL, Caldamone AA, Gauderer M, Hampel N, Wesselhoeft CW, Elder JS. Infected urachal cysts: a review of 10 cases. J Urol. 1988;140(2):375-378.

8. Gómez Parada J, Puyol Pallas JM. Quiste infectado de uraco: a propósito de un nuevo caso. Arch Esp Urol. 2001;54(7):722-725.

9. Marmol Navarro S, Guadalajara Jurado J, Cancelo Suarez P, Gil de la Puente J, Rajab R, Parra Mountaner LE. Quiste piouracal. Arch Esp Urol. 2001;45(10):1034-1036.

10. Burgués Gasión JP, Domínguez Hinarejos C, Serrano Durbá A, Estornell Moragues F, Martínez Verduch M, García Ibarra F. Divertículos de uraco. Diagnóstico y tratamiento. Arch Esp Urol. 2002;55(3):285-291.

11. Chauvin N, Domachowske JB. Infected urachal cyst presenting as fever of unknown origin. Clin Pediatr. 2005;44 (1):85-87.

12. Allen JW, Song J, Velcek FT. Acute presentation of infected urachal cysts: case report and review of diagnosis and therapeutic interventions. Pediatr Emerg Care. 2004;20(2): 108-111.

13. MacNeily AE, Koleilat N, Kiruluta HG, Homsy YL. Urachal abscesses: protean manifestations their recognition, and management. Urology. 1992;40:530-535.

14. Guarnaccia SP, Mullins TL, Sant GR. Infected Urachal Cysts. Urology. 1990;36(1):61-65.

15. Mesrobian HG, Zacharias A, Balcom AH, Cohen RD. Ten years of experience with isolated urachal anomalies in children. J Urol 1997;158(3 Pt 2):1316-1318.

16. Pesce C, Costa L, Musi L, Campobasso P, Zimbardo L. Relevance of infection in children with urachal cysts. Eur Urol. 2000;38(4):457-460.

17. Luo CC, Huang CS, Wu WC, Chu SM, Chao HC. An unusual presentation of an infected urachal cyst: case report and review of the literature. Eur J Pediatr. 2004;163(4-5): 268-269.

18. Newman BM, Karp MP, Jewett TC, Cooney DR. Advances in the management of infected urachal cysts. J Pediatr Surg. 1986;21:1051-1054.

19. Donate Moreno MJ, Giménez Bachs JM, Salinas Sánchez AS, Lorenzo Romero JG, Segura Martín M, Hernández Millán I et al. Patología del uraco: revisión de conjunto y presentación de tres casos. Actas Urol Esp. 2005;29(3): 332-336.

Dr. V. Gimeno Argente

E-mail: vigiar71@hotmail.com

(Trabajo recibido el 26 de marzo de 2006) 